\title{
Statistical Feature Analysis of EEG Signals for Calmness Index Establishment
}

\section{Siti Armiza Mohd Aris", Siti Zura A.Jalil, Nurul Aini Bani, Hazilah Mad Kaidi, Mohd Nabil Muhtazaruddin}

\author{
${ }^{1}$ Razak Faculty of Technology and Informatics, \\ Universiti Teknologi Malaysia, Jalan Sultan Yahya Petra, Kuala Lumpur, 54100, MALAYSIA \\ *Corresponding Author \\ DOI: https://doi.org/10.30880/ijie.2018.10.07.003 \\ Received 25 October 2018; Accepted 15 November 2018; Available online 30 November 2018
}

\begin{abstract}
Electroencephalographic (EEG) signals are very closely related to psychophysiological. The EEG signals displayed few responses which can be categorized. This article discussed the use of statistics over the EEG features which confirm the different mental characteristics. Two different type of stimulus was given named as relaxed state and non-relaxed state. Asymmetry index was computed as the EEG features via the alpha waves and was extracted during the relaxed state and the non-relaxed state. The EEG features were clustered to a group of three, four and five using Fuzzy C-Means. During the relaxed state, the alpha wave showed a higher response as compared to the nonrelaxed state. This is observed by using the mean relative energy between the relaxed state and non-relaxed state. To ensure which EEG features in the clusters showed a significant difference, $\mathrm{p}<.05$, a statistical test was used. Wilcoxon Signed Ranks test is the best-statistical test to verify the selected clusters as it is suitable to analyze the small sample of data. Wilcoxon Signed Ranks test used a hypothesis testing which using the same method as paired sample t-test. The advantage in using Wilcoxon Signed Ranks test is that, it uses the median to get the difference between two samples of data. Analytical results showed that the data features of four clusters and three clusters give a significant difference, thus the obtained results can be used to further up the study. The Wilcoxon Signed Ranks test results confirmed that the proposed technique has potential in establishing the calmness index.
\end{abstract}

Keywords: EEG, Asymmetry Index, Statistical Features, FCM, Wilcoxon Signed Ranks, Calmness Index

\section{Introduction}

The human brain, with an unimposing exterior, contains a wealth of information which can be discovered and interpreted. With an advancement in signal processing techniques, many discoveries had been achieved by exploring the brain signals. In addition to the signal processing method, the data should also be interpreted statistically. The used of statistical analysis is not limited, can be used to obtain the difference between data, comparing the strength of data and also for data validation. Miller has explained how statistics had helped many medical researchers in detecting diseases and making conclusions on the possibility of the disease occurring (Miller, 1979). This is also supported by other researchers who provide statistical analysis in their work (Locatelli et al., 1991; Maris \& Oostenveld, 2007; Ogawa, Karungaru, Mitsukura, Fukumi, \& Akamatsu, 2006). This undoubtedly explains why statistic is important in biosciences research which has become important as it is a part of the quantitative approach to knowledge.

A statistical test is usually used by researchers to discover variables that differ significantly between groups (Fielding, 2007) and also could lead them to significant results (Cohen, 1988). In data analysis, there are two types of data test; parametric tests and non-parametric tests. To choose a parametric test, the data must fall into a normal distribution. However, to achieve normal distribution for all data type is quite challenging. Thus, an alternative to the parametric test is the non-parametric test. Normally, data which related to human will fall under non-parametric tests (Tomkins \& Hall, 
2006). The sample size has implications to any of the statistical techniques that will be chosen such as in rejecting the null hypothesis (power) (Cohen, 1988). Small sample sizes are able to achieve adequate power if the non-parametric is chosen (Tomkins \& Hall, 2006; Wilcoxon, 1945). Such as for the small sample size, Wilcoxon signed rank test can be used which is one of the examples of non-parametric test.

Another guideline that one should consider when selecting a non-parametric test that, it is comparable to a parametric test. The Wilcoxon signed rank test is an alternative test to the parametric paired sample t-test (Helen, 2008; Pallant, 2007; Sullivan, 2004). Some researchers choose to use the non-parametric test, especially in smaller data samples, which seldom falls into the normal distribution or when the homogeneity of variance is presented (Helen, 2008). In 1945, Wilcoxon initiated a study on the comparison of treatments by introducing the signed-rank and the rank sum statistics (Wilcoxon, 1945). Wilcoxon proposed the statistical test methods to evaluate data that come from a non-normal distribution or small data size in ensuring the normality. Wilcoxon signed rank test provides a powerful test in perceiving a difference between either two small samples or large samples (Bellera, Julien, \& Hanley, 2010). The selection of the statistical test was also based on the sample size $(N)$.

Many researchers have selected Wilcoxon signed rank test as their statistical tools. Some studies were conducted by the same researchers with the aim to demonstrate the differences between the EEG mental tasks via different feature extractions, namely approximate entropy (ApEn) (Jann, Koenig, Dierks, Boesch, \& Federspiel, 2010), detrended fluctuation analysis (DFA) (Vega \& Fernandez, 2012b), and phase locking value (PLV) (Vega \& Fernandez, 2012a). Wilcoxon signed rank test appropriately proved there is a significant difference between the mental tasks chosen in the experiments. There is another study on EEG related mental tasks which applied Wilcoxon signed rank test in their statistical analysis and using Wilcoxon test; significant differences were obtained through the sample entropy and average power in the comparison of the various mental tasks (Amin, Malik, Badruddin, \& Chooi, 2013).

In the review, it was acknowledged that the alpha wave could be a good indicator of relaxation (Allen, Urry, Hitt, \& Coan, 2004; Maxwell \& Davidson, 2007). Alpha wave is also clearly seen during eyes closed and will be diminished directly after an individual open the eyes. In association with the alpha wave, an asymmetry index (AsI) could be extracted to become an EEG feature. The AsI is able to produce unique values which can differentiate one individual from another. In practice, the AsI is a differentiation between two indices, which can have either a negative or positive value or sometimes zero, regardless of the state of the brain. Therefore, it is possible to group similar behaviour even with different brain states. Consequently, FCM can be used to facilitate in grouping the asymmetry index which has approximate behaviour that lies within the data features. One of the advantages of using FCM is that it allows one member of the data to belong to two or more clusters specified by a membership grade and this would give some freedom during the data grouping. The grouped data selected by FCM need to be verified, as this is also one way to validate the clustering made. To support the findings, the statistical analysis needs to be included in the study.

\section{Methodology}

The flow chart in Fig. 1 provides the flow of experiments methodically prepared for the study. The work comprises of the collection of EEG data during relaxed and non-relaxed conditions, extractions of EEG features, clustering of the EEG features, computation of mean relative energy and lastly statistical analysis to validate the EEG features for EEG calmness index.

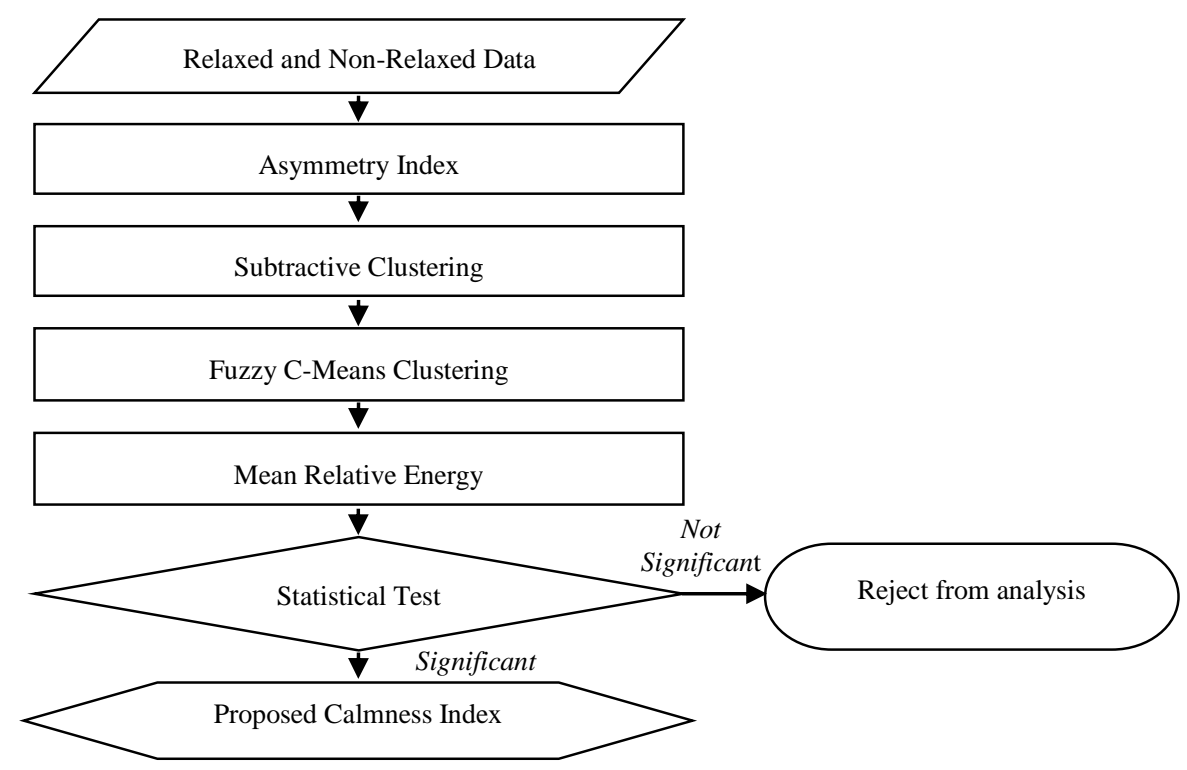

Fig. 1 - Study flow 


\subsection{Experiment Protocol}

The subjects involved in this study were 43 students from the university, aged between 22 and 24 years old. They are all right-handed and are not under any medications. Subjects have been explained about the experiments, and they have agreed to participate in the study voluntarily. In this study, the EEG data were recorded at the pre-frontal, Fp1 and Fp2 and because of the connections is the bipolar type, each channel was paired with the left and right earlobes respectively. The electrodes placement is shown in Fig. 2.

\section{Anterior}

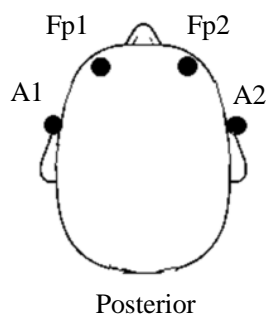

Fig. 2 - Electrodes placement

In Table 1 shows the relaxed state data collection procedure. Relaxed state is synonymous with the resting state. During the data recording, the participants rest on a comfortable chair in a soundless room with dim lights and were asked to close their eyes during the EEG recording. Another type of brain stimulus used in the study was the mental task test. The participants were requested to answer to a non-verbal and logical thinking task test which the idea was taken from the Raven Test. Questions are designed so that users can interact directly with the computer. The purpose of mental task test is to engage the subjects' brain into a working state, so that a contrary condition than the relaxed state is achieved.

Table 1 - Experiment procedure

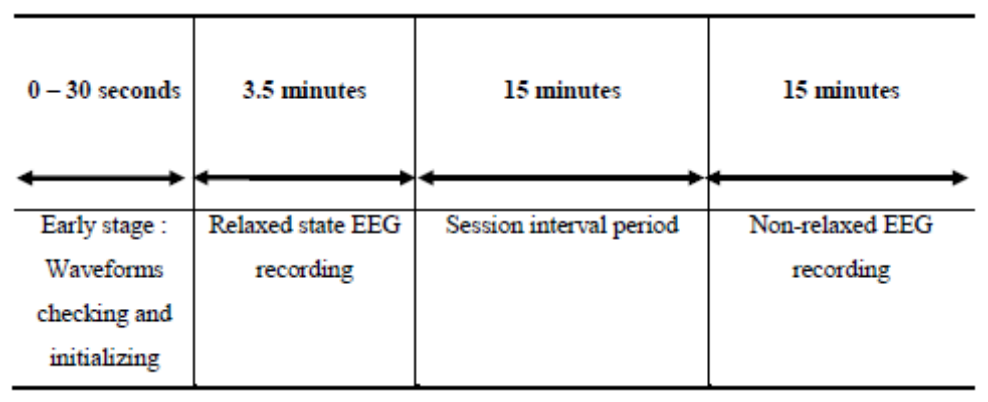

The raw EEG data consists of 86 data (relaxed state and non-relaxed state) were pre-processed using Fast Fourier Transform (FFT) and Hamming window with $50 \%$ overlapping. Following with the computation of Discrete Fourier Transform (DFT) before ESD was calculated. During the EEG data pre-processing, the EEG data were processed only for three minutes in all conditions. The acquisition tools used in the study was the g.MOBIlab+, with the sampling rate of $256 \mathrm{~Hz}$.

\subsection{EEG Features}

In the study, the asymmetry index (AsI) was computed from the relaxed and non-relaxed states as the EEG data features. Asymmetry index was selected as the EEG features and converted using Equation (1), where AsI is the difference of natural-log of power at the right hemisphere $\operatorname{Po}\left(F p_{2}\right)$ and at the left hemisphere $\operatorname{Po}\left(F p_{1}\right)$.

$$
A s I=\ln \left[P o\left(F p_{2}\right)\right]-\ln \left[P o\left(F p_{1}\right)\right]
$$

\subsection{Clustering}

In FCM was selected in the previous study to cluster all data points into the same group with approximate behaviour. The process starts with transforming the data into membership grades and initialized into appropriate matrix, U, where $\|$. $\|$ is the Euclidean distance. Basic FCM algorithm is given in (2).

$$
J=\sum_{k=1}^{n} \sum_{i=1}^{c} \mu_{i k}^{m}\left\|x_{k}-v_{i}\right\|^{2}
$$


where $\mathrm{n}$ is the total number of data points, $\mathrm{c}$ is the number of clusters, $x k$ is the $k$ th data point, $v i$ is the $i$ th cluster center and $m$ is a constant which is greater than 1 .

One of the drawbacks of FCM is to determine the number of clusters. Thus, it needs another tool to identify the suitable clusters center within the given data. One of the tools is subtractive clustering (SC). The SC method also improves the criterion for accepting and rejecting cluster centers. SC does not require the number of cluster to be pre-specified. They are processed in off-line and the same data samples can be processed many times. The number of clusters generated by the SC can be used as the predetermined number for FCM clusters (Alata, Molhim, \& Ramini, 2008; Wen-Yuan, Chun-Jing, Bao-Wen, Yan, \& Shu-Fen, 2003).

\subsection{Mean Relative Energy}

The energy content per group clustered by FCM was revealed using mean relative energy. It is one of the methods to compare the performance of each clusters. Mean relative energy was the averaged value computed from total subjects. In the study, the mean relative energy for each cluster during relaxed state and non-relaxed state was computed and plotted.

Since the Fp1 and Fp2 were used in the study, the relative energy for alpha band $E_{R E}$ can be determined using

$$
E_{R E}=\frac{\left(E_{\text {diff }} \times E_{\Delta}\right)}{E_{T}} \times 100 \%
$$

where, $E_{\Delta}$ is the total energy of the selected EEG bands, such as in the study is alpha. The total energy $E_{T}$ is the sum of the whole energy components found in the given frequency bands as,

$$
E_{T}=E_{\text {delta }}+E_{\text {theta }}+E_{\text {alpha }}+E_{\text {beta }}
$$

and, $E_{\text {diff }}$ is the difference between Fp1 and Fp2. The mean relative energy, $E_{M R E}$, can be computed using,

$$
E_{M R E} \frac{\sum_{1}^{n} E_{R E}}{S_{n}}
$$

is the value of average relative energy from the total number of subjects $S n$, with $n$ is number of subjects.

\subsection{Wilcoxon Signed Ranks Test}

The Wilcoxon signed ranks test is a nonparametric hypothesis test in which the original data are converted into ranks. The Wilcoxon signed ranks test can analyses for a single population median or matched-pair data from two dependent samples. To execute the test, selected data must be random and symmetric distribution. Wilcoxon Signed Ranks test was chosen to further analyses a small sample of data [8]. Wilcoxon Signed Ranks test used a hypothesis testing which using the same method as paired sample t-test. The advantage in using Wilcoxon Signed Ranks test is that, it uses the median to get the difference between two samples of data. Thus, the analysis will not be biased to the number of data available and suitable for the non-normal type of data.

Wilcoxon Signed Ranks test was chosen in the analysis to test the significant difference between two different psycho-psychological states selected by FCM. This test is an alternative to paired sample t-test for non-normal data distributions. The hypothesis testing for sample size less than 30 data features for each cluster of two psychopsychological states are;

$$
\begin{aligned}
& H_{0}: m_{D}=0 \\
& H_{1}: m_{D} \neq 0
\end{aligned}
$$

The null hypothesis was fixed such that the population median difference between pairs, $m_{D}$ is equal to zero. On the other hand, the alternative hypothesis was fixed such that the population median difference between pairs, $m_{D}$ is not equal to zero. Hence, this is a two-tailed test with target $p$-value must be less than 0.05 to achieve significance difference.

In order to make the results more powerful, it is worthwhile to achieve the efficiency which is comparable to that of the parametric test. Such as the Wilcoxon signed ranks test, which represent the non-parametric test, the matched efficiency is $95.5 \%$ which is comparable to the parametric test, paired sample t-test (Sullivan, 2004). The Wilcoxon signed ranks test is also parametric equivalent to Student's $t$-test, $t$-test for matched pairs, $t$-test for paired samples or $t$ test for dependent samples (Corder \& Foreman, 2009). Basically, Wilcoxon Signed Ranks test is testing hypothesis on median. The values are changed into positive ranks and negative ranks, while any zero difference are eliminated from consideration.

The sum of $W+$ and sum of $W$ - are compared in order to make the selection of the smaller value possible. The $n u l l$ hypothesis has been set that the median is zero. Wilcoxon has proven that the distributions of his examined data have 
changed to normal distribution by using his method (Wilcoxon, 1947). The Wilcoxon Signed Ranks test can be computed using SPSS.

$$
\begin{aligned}
& W^{+}=\sum R_{+} \\
& W^{-}=\sum R_{-}
\end{aligned}
$$

\section{Results and Discussion}

This section discusses the research findings before the calmness index is selected. This section starts with the conversion results of the EEG signal to the asymmetry index. Then, the FCM results which classify the EEG features into the similar group. Followed by the relative mean energy results in comparing the relaxed state and the non-relaxed states and lastly are the results of a statistical test using the Wilcoxon Signed Ranks test.

\subsection{Asymmetry Index}

The relaxed state and the non-relaxed state AsI were then separately plotted. This was done in order to scrutinize the data behaviour by means of simple linear regression. From the plotted regression line in Fig. 3 showed that the AsI values during relaxed state were biased towards the positive. The plotted line which representing the trend of the 43 subjects during relaxed state was linearly increased. When the AsI value closes to the positive value, it describes greater right frontal was obtained. It can also be described as the left hemisphere activity was higher than the right.

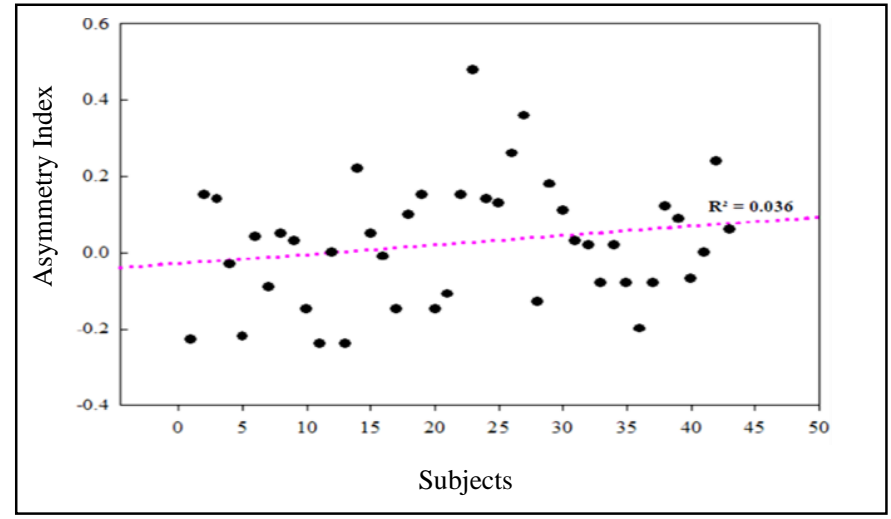

Fig. 3 - EEG features during relaxed state

Fig. 4 shows the response of AsI values from 43 subjects during non-relaxed state. The trend of the linear line for this group was biased toward the negative and this was contradicted to the relaxed state. The results show that during the non-relaxed state, the left frontal was higher than the right frontal which also indicated that the right hemisphere was responding to the given stimulus.

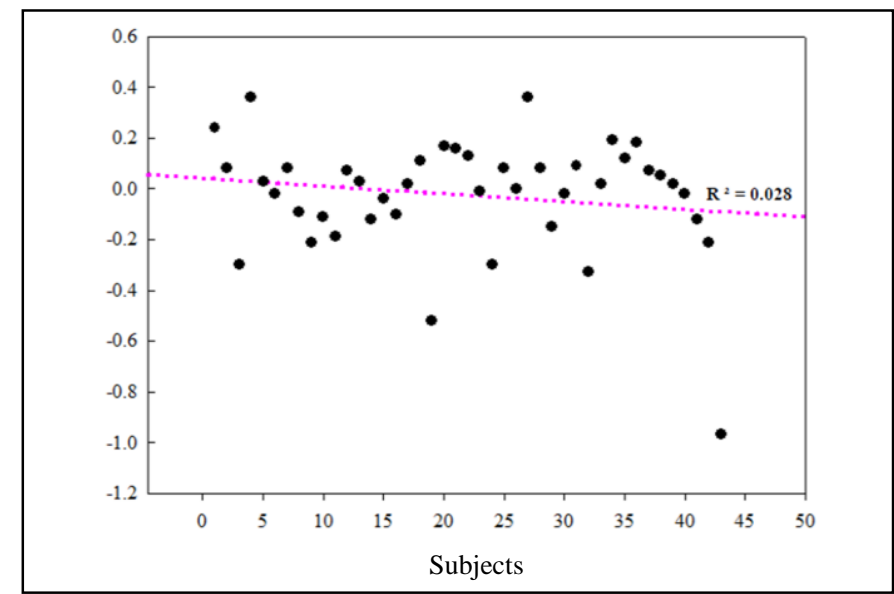

Fig. 4 - EEG features during non-relaxed state

\subsection{Subtractive Clustering and Fuzzy C-Means Clustering}

Subtractive clustering (SC) was utilized in the study to estimate the cluster centers accessible in the EEG data. The generated cluster center is shown in Fig. 5. Thus, in the study there are five possible accessible cluster centers generated 
from the SC process. The resulting of the cluster center number was used as the pre-set number in the computation of FCM. Results from the SC analysis were used as the pre-set number for the FCM. In this section, the results of the FCM clustering were divided into three pre-set numbers.

Given from the SC results, the maximum number of clusters that were visible within the EEG data were five, thus the pre-set number of FCM was divided into five, four and three number of pre-sets. The purpose of separating the preset numbers which was to be analysed by the FCM is to optimize the number of clusters that are appropriate to be used in the EEG calmness index establishment.

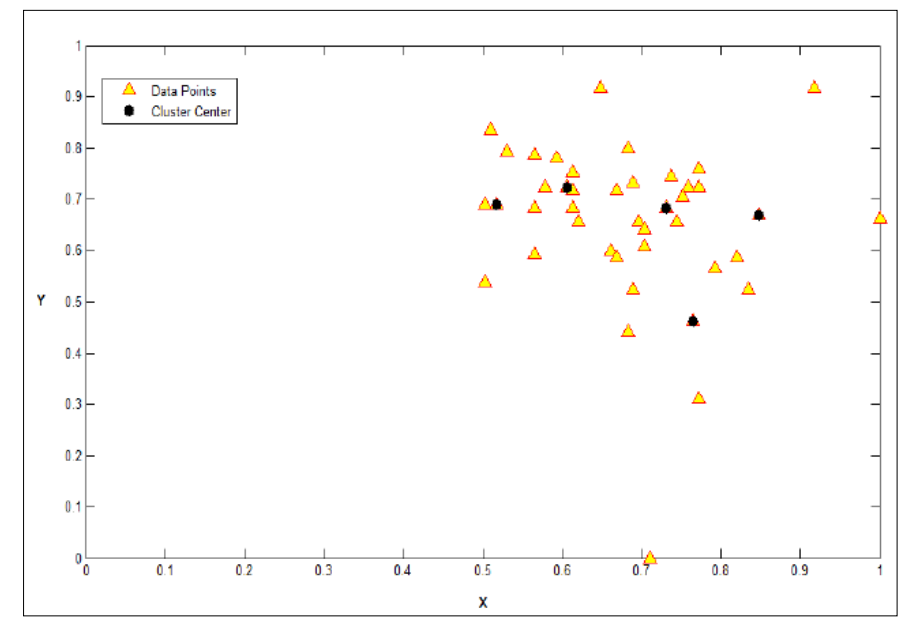

Fig. 5 - Pre-determined cluster centers

The FCM algorithm has been carried out several times to reach the best cluster center that fits the data. The selection was based on the minimum iterations achieved at the end of the algorithm and the position of the cluster center which appears at the same place after several runs. The selected data points to be in the same group were marked with the similar symbol according to the groups as 132 shown in Fig. 6, Fig. 7 and Fig. 8 for five clusters, four clusters and three clusters respectively.

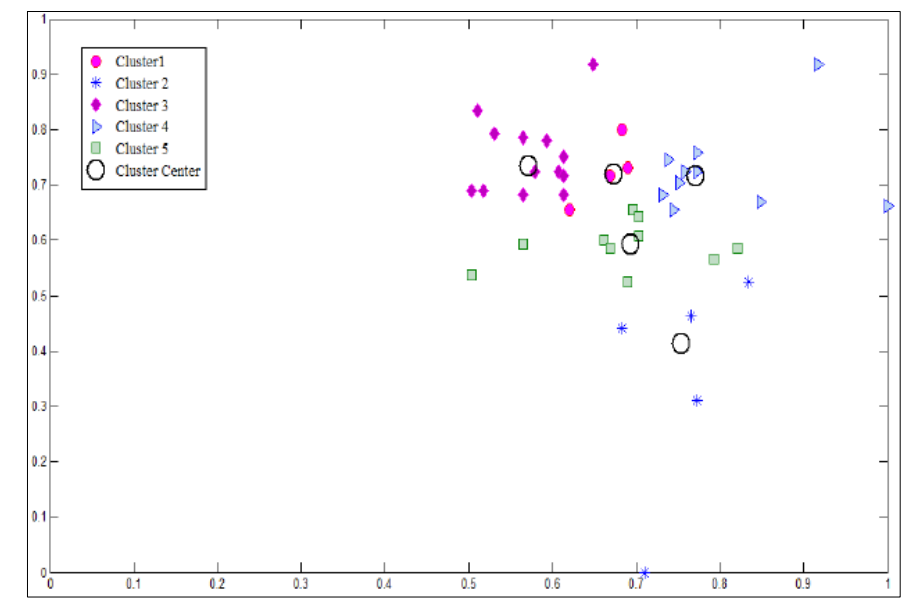

Fig. 6 - FCM clustering - 5 clusters

The selected data points to be in the same group based on the nearest distance from the cluster center and were marked with a similar symbol according to the groups. Each cluster's center which also known as the centroid, is the average distance from the total data points within the same group. As shown in Fig. 6 for the five clusters, different symbols have been generated that follow their respective cluster. Each group has its own centroid and the same applies to four clusters and three clusters demonstrated in Fig. 7 and Fig. 8. The computed centroid was also weighted by their degree of belonging to the cluster. 


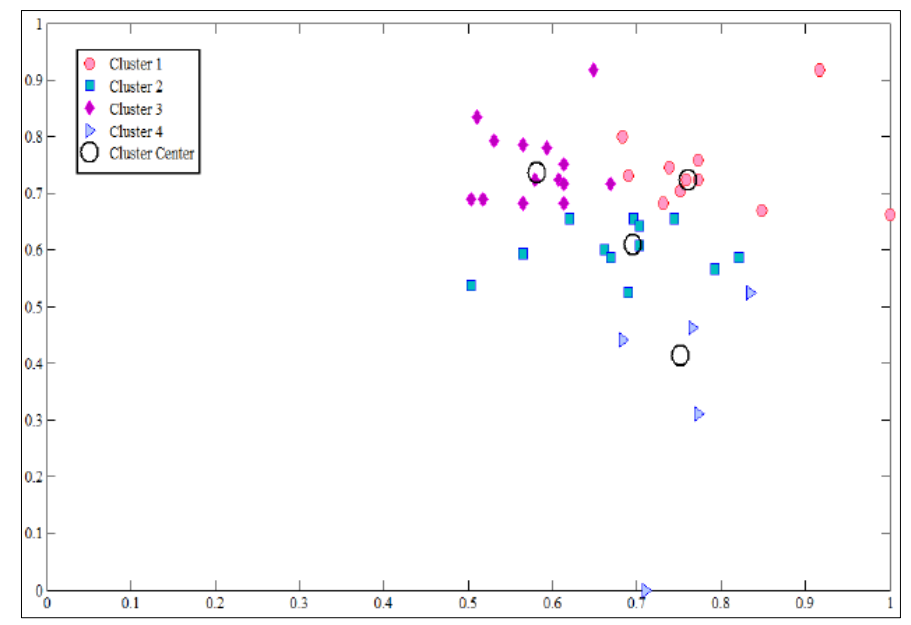

Fig. 7 - FCM clustering -4 clusters

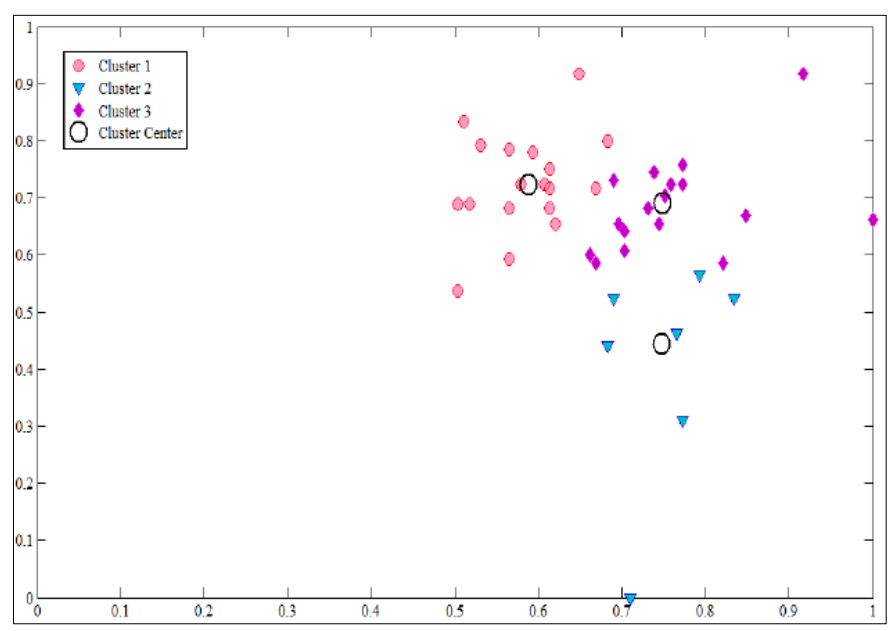

Fig. 8 - FCM clustering -3 clusters

The results of FCM clustering for the five clusters, the four clusters and the three clusters were used to attain results in the next section. The subsequent section is the results which were to observe the ability of FCM in selecting the EEG data that represent the same behaviour into a cluster. Hence, to observe the difference the data was expounded upon again in the next section in a form of mean relative energy.

\subsection{Mean Relative Energy}

Computation of relative energy was applied to each cluster consisting of the EEG data features during relaxed state and non-relaxed state. EEG data that has been computed into relative energy were summed and averaged by the number of subjects for each group during relaxed state and non-relaxed state. Thus, the plotted results were based from the mean value of the totalled relative energy. Each cluster which comprises of two EEG states (relaxed state and non-relaxed state) were plotted to observe its response. Fig. 9, Fig. 10 and Fig. 11 shows the clustering of features for five clusters, four clusters and three clusters, respectively.

From all the results attained, the relaxed state energy was proven has achieved higher energy level than that of the non-relaxed state. These findings had validated that the search of index has supported the quest on the calmness index. This section, assumed as pre-index establishment, has confirmed that all values of the mean relative energy were positive which ultimately declared that the energy during relaxed state is higher than that of non-relaxed state. The results of the mean relative energy showed that, all three sets of clusters can be proposed as calmness index as regards to the significant results achieved. However, it was not sufficient to merely get the difference between the relaxed state and the non-relaxed state. Thus, statistical analysis was carried out to decide which numbers of clusters are suitable to be proposed as the calmness index. The results were discussed in the next section. 


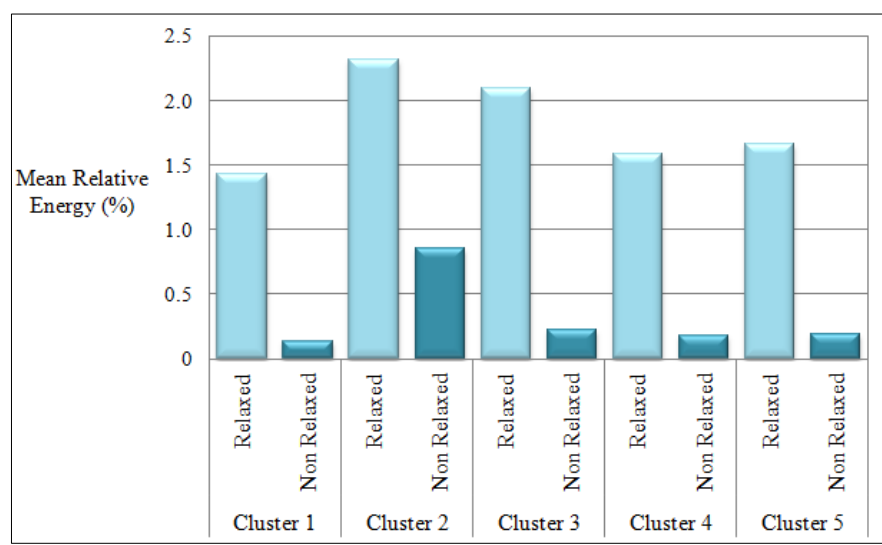

Fig. 9 - Mean relative energy patterns of five clusters

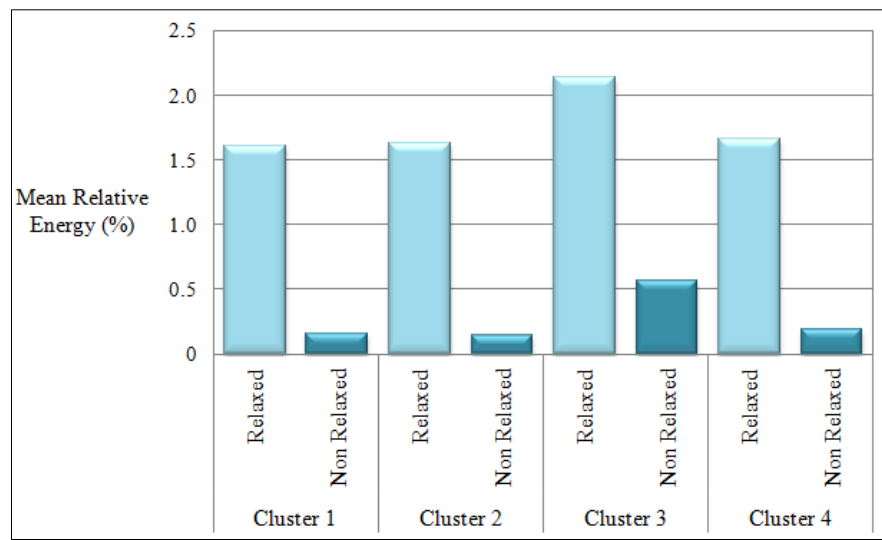

Fig. 10 - Mean relative energy patterns of four clusters

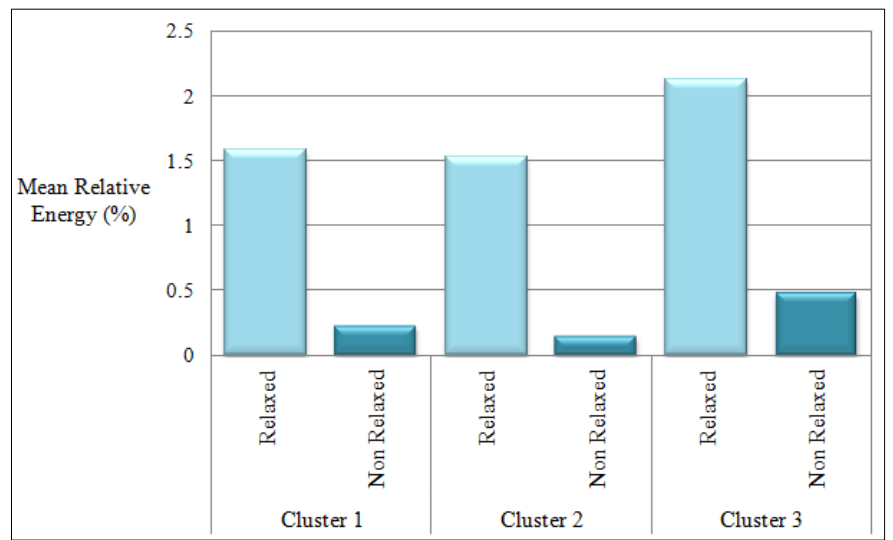

Fig. 11 - Mean relative energy patterns of three clusters

\subsection{Wilcoxon Signed Ranks Test}

In addition, to find the difference between the two psycho-psychological states, a proper statistical test should be selected. EEG data that have been tested using numerical tests showed that most of the data was not normal. Therefore, a comparable test with paired sample t-test for non-normal data is the Wilcoxon Signed Ranks test. For that reason, Wilcoxon Signed Ranks test was chosen to further analyse a small sample of data (Wilcoxon, 1945). The output of SPSS for Wilcoxon Signed Rank Test of five clusters, four clusters and three clusters were discussed in this section.

The data within the clusters were analysed using Wilcoxon Signed Rank test with targeted p-value was set to be less than 0.05 to achieve significant difference. The output of SPSS for Wilcoxon Signed Rank Test of five clusters is shown in Table 2. The significant of the findings were completed with the analysis of the p-value and the Z-value. The list of the clusters was the Cluster 1 which has achieved the $\mathrm{p}=.008$ and the $\mathrm{Z}=-2.666$. The next cluster was the Cluster 3 , with the $p=.003$ and the $Z=-2.934$. Another significant cluster was the Cluster 4 , with the $p=.005$ and the $Z=-2.803$. Lastly was the Cluster 5, with the $\mathrm{p}=.028$ and the $\mathrm{Z}=-2.201$. 
Table 2 - Wilcoxon signed ranks test for five clusters

\begin{tabular}{|c|c|c|}
\hline & \multicolumn{2}{|c|}{ Value } \\
\hline \multirow{2}{*}{ Cluster 1 (Relaxed - NonRelaxed) } & Z & -2.666 \\
\hline & $\begin{array}{l}\text { Asymp. Sig. } \\
\text { (2-tailed) }\end{array}$ & .008 \\
\hline \multirow{2}{*}{ Cluster 2 (Relaxed - NonRelaxed) } & Z & -1.352 \\
\hline & $\begin{array}{l}\text { Asymp. Sig. } \\
\text { (2-tailed) }\end{array}$ & .176 \\
\hline \multirow{2}{*}{ Cluster $\mathbf{3}$ (Relaxed - NonRelaxed) } & Z & -2.934 \\
\hline & $\begin{array}{l}\text { Asymp. Sig. } \\
\text { (2-tailed) }\end{array}$ & .003 \\
\hline \multirow{2}{*}{ Cluster 4 (Relaxed - NonRelaxed) } & z & -2.803 \\
\hline & $\begin{array}{l}\text { Asymp. Sig. } \\
\text { (2-tailed) }\end{array}$ & .005 \\
\hline \multirow{2}{*}{ Cluster 5 (Relaxed - NonRelaxed) } & Z & -2.201 \\
\hline & $\begin{array}{l}\text { Asymp. Sig. } \\
\text { (2-tailed) }\end{array}$ & .028 \\
\hline
\end{tabular}

Wilcoxon Signed Rank test was also run to four clusters to determine the differences between the relaxed state and the non-relaxed state as shown in Table 3. The results obtained in Cluster 1 showed that the $\mathrm{p}=.002$ and $\mathrm{Z}=-3.059$. From the test results of Cluster 2, a statistically significant was also achieved with $p=.03$ and $Z=-2.934$. Similar findings were also obtained in Cluster 3 with $p=.011$ and $Z=-2.542$ which indicated that the result was statistically significant. Lastly, for Cluster 4, the Wilcoxon Signed Rank test has successfully achieved $p=.28$ and Z $=-2.201$ which also indicates that the test has achieved statistically significant. The above discussed results have demonstrated that all clusters successfully differentiate between the relaxed state and the non-relaxed state which contained within all clusters. The significant results obtained have enabled the four clusters to be proposed as the calmness index which will be determined in the next study.

Table 3 - Wilcoxon signed ranks test for four clusters

\begin{tabular}{|c|c|c|}
\hline & \multicolumn{2}{|c|}{ Value } \\
\hline \multirow{2}{*}{ Cluster 1 (Relaxed - NonRelaxed) } & Z & -3.059 \\
\hline & $\begin{array}{c}\text { Asymp. Sig. } \\
\text { (2-tailed) }\end{array}$ & .002 \\
\hline \multirow{2}{*}{ Cluster 2 (Relaxed - NonRelaxed) } & Z & -2.934 \\
\hline & $\begin{array}{l}\text { Asymp. Sig. } \\
\text { (2-tailed) }\end{array}$ & .003 \\
\hline \multirow{2}{*}{ Cluster 3 (Relaxed - NonRelaxed) } & Z & -2.542 \\
\hline & $\begin{array}{l}\text { Asymp. Sig. } \\
\text { (2-tailed) }\end{array}$ & .011 \\
\hline \multirow{2}{*}{ Cluster 4 (Relaxed - NonRelaxed) } & z & -2.201 \\
\hline & $\begin{array}{l}\text { Asymp. Sig. } \\
\text { (2-tailed) }\end{array}$ & .028 \\
\hline
\end{tabular}

The last set of clusters that was also analysed by the Wilcoxon Signed Rank test. Table III showed the result of each cluster generated by the Wilcoxon Signed Rank test. All of the groups have successfully accomplished significant results. This was evidenced by achieving $\mathrm{p}=.012$ and $\mathrm{Z}=-2.524$ for Cluster 1 . Followed by $\mathrm{p}=0$ and $\mathrm{Z}=-3.621$ for $\mathrm{Cluster} 2$ and lastly for Cluster $3, p=0.002$ and $Z=-3.070$. test was the three clusters. All of the $p$-values and the $Z$-values obtained from the Wilcoxon Signed Ranks test for three clusters implied that there were significant differences between the relaxed state and non-state relaxed which contained in each cluster. Thus, the three clusters were also feasible to be used as an index of calmness besides the four clusters 
Table 4 - Wilcoxon signed ranks test for three clusters

\begin{tabular}{|c|c|c|}
\hline \multirow{2}{*}{ Cluster 1 (Relaxed - NonRelaxed) } & \multicolumn{2}{|c|}{ Value } \\
\cline { 2 - 3 } & $\begin{array}{c}\text { Asymp. Sig. } \\
\text { (2-tailed) }\end{array}$ & .012 \\
\hline \multirow{2}{*}{ Cluster 2 (Relaxed - NonRelaxed) } & $Z$ & -3.524 \\
\cline { 2 - 3 } & $\begin{array}{c}\text { Asymp. Sig. } \\
\text { (2-tailed) }\end{array}$ & .000 \\
\hline \multirow{2}{*}{ Cluster 3 (Relaxed - NonRelaxed) } & Z & -3.070 \\
\cline { 2 - 3 } & Asymp. Sig. & .002 \\
\hline
\end{tabular}

\section{Conclusion}

In this study, the collected EEG data were pre-processed and computed before it was used as the EEG features. The pre-processed EEG signals were confirmed its quality to ensure the received EEG signals were highly discriminated between two conditions, namely EEG relaxed state and EEG non-relaxed state. Both pre-processed EEG signals states were analysed and computed into the EEG features, asymmetry index.

The FCM was used in the study as to segregate the EEG data which shows the same behaviour. From the results, the lesser the cluster centers, the farther the distances between the clusters centers and the lessen counts needed for the algorithm to converge. The reason for separating it into three division of pre-set number is to optimize the EEG data into few sets of clusters before it was proposed into the EEG calmness index. The aim was to have more than two different groups as at present, studies only focusing on two different indices (Hinrikus et al., 2009; Musha, Kimura, Kaneko, Nishida, \& Sekine, 2000; Suhhova et al., 2013; Teplan, Krakovská, \& Štolc, 2009). Thus, the two clusters analysis was not included in the study.

The results of the EEG data that were selected by FCM fall into several clusters have been unravelled and identified in this section by using the computation of relative energy. Each data point that was chosen by FCM represents the data features during relaxed state and non-relaxed state. Mean relative energy is the average of the data features per cluster for relaxed state and non-relaxed state. The results of mean relative energy show the behaviour of the EEG signals in each cluster for the relaxed state is higher than the non-relaxed state. In this case, the mean relative energy for each cluster during relaxed state and non-relaxed state was computed and plotted. The results were arranged for five clusters, four clusters, and three clusters. The pattern for each cluster types showed that during the relaxed state, the alpha waves were higher than the non-relaxed state. From the mean relative unique patterns, a robust and reliable calmness index were figured out which by using a statistical test, Wilcoxon Signed Ranks test.

Wilcoxon Signed Ranks test uses the median to get the difference between the two samples. Thus, the analysis will not be biased to the number of data available and suitable for the non-normal type of data. From the overall Wilcoxon Signed Ranks test results show that for five clusters, there was non-significant result in cluster 2 . Thus, five clusters could not be proposed to be used as the calmness index. The three and the four cluster types will be proposed in the future study.

The significant finding in the study was fully utilized from the ability of the EEG signals in differentiating several characteristics. The alpha wave which closely related to calmness was chosen to establish the index. The alpha waves were filtered and computed during relaxed state and non-relaxed state. The plotted mean relative energy of the alpha waves showed that each cluster portrayed a unique response between two EEG states. All cluster showed that during the relaxed state, the alpha waves response was higher than during the non-relaxed state. The statistical test was used to confirm the significant difference, and it is proven that the EEG signals can bring so much information from the brain.

\section{Acknowledgement}

The authors would like to thank the Razak Faculty of Technology and Informatics, Universiti Teknologi Malaysia for the sponsorship of the study under research grant NO: R.K130000.7340.4J301 and also to all collaborating partners.

\section{References}

[1] Alata, M., Molhim, M., \& Ramini, A. (2008). Optimizing of fuzzy c-means clustering algorithm using GA. World Academy of Science, Engineering and Technology, 1(5), 224-229.

[2] Allen, J. J. B., Urry, H. L., Hitt, S. K., \& Coan, J. A. (2004). The stability of resting frontal electroencephalographic asymmetry in depression. Psychophysiology, 41(2), 269-280. 
[3] Amin, H. U., Malik, A. S., Badruddin, N., \& Chooi, W.-T. (2013). EEG mean power and complexity analysis during complex mental task. Paper presented at the 2013 ICME International Conference on Complex Medical Engineering (CME).

[4] Bellera, C. A., Julien, M., \& Hanley, J. A. (2010). Normal Approximations to the Distributions of the Wilcoxon Statistics: Accurate to What N? Graphical Insights. Journal of Statistics Education, 18(2), 1-17.

[5] Cohen, J. (1988). Statistical power analysis for the behavioral sciences: Lawrence Erlbaum Associates Inc.

[6] Corder, G. W., \& Foreman, D. I. (2009). Nonparametric Statistics for Non-Statisticians: A Step-by-Step Approach: John Wiley \& Sons, Inc.

[7] Fielding, A. (2007). Cluster and classification techniques for the biosciences: Cambridge Univ Pr.

[8] Helen, G. (2008). Understanding Research Methods and Statistics in Psychology. London: SAGE Publications Inc.

[9] Hinrikus, H., Suhhova, A., Bachmann, M., Aadamsoo, K., VÃuhma, Ã., Lass, J., \& Tuulik, V. (2009). Electroencephalographic spectral asymmetry index for detection of depression. Medical and Biological Engineering and Computing, 47(12), 1291-1299.

[10] Jann, K., Koenig, T., Dierks, T., Boesch, C., \& Federspiel, A. (2010). Association of individual resting state EEG alpha frequency and cerebral blood flow. NeuroImage, 51(1), 365-372.

[11] Locatelli, M., Gambini, O., Colombo, C., Canger, R., Beltrami, M., \& Scarone, S. (1991). A statistical approach to computerized EEG: Preliminary data on control subjects and epileptic patients. Brain Topography, 3(4), 401-406.

[12] Maris, E., \& Oostenveld, R. (2007). Nonparametric statistical testing of EEG- and MEG-data. Journal of Neuroscience Methods, 164(1), 177-190.

[13] Maxwell, J. S., \& Davidson, R. J. (2007). Emotion as Motion: Asymmetries in Approach and Avoidant Actions. Psychological Science, 18(12), 1113-1119.

[14] Miller, R. W. (1979). Statistics and Biomedical Research. Environmental Health Perspectives, 32, 17-18.

[15] Musha, T., Kimura, S., Kaneko, K. I., Nishida, K., \& Sekine, K. (2000). Emotion spectrum analysis method (ESAM) for monitoring the effects of art therapy applied on demented patients. CyberPsychology \& Behavior, $3(3), 441-446$.

[16] Ogawa, T., Karungaru, S., Mitsukura, Y., Fukumi, M., \& Akamatsu, N. (2006). Feature Extraction in Listening to Music Using Statistical Analystis of the EEG. Paper presented at the 2006 International Joint Conference of SICE-ICASE.

[17] Pallant, J. F. (2007). SPSS Survival Manual: a step by step guide to data (3rd ed.). Australia: Allen \& Unwin.

[18] Suhhova, A., Bachmann, M., Lass, J., Aadamsoo, K., VÃ $\mu$ hma, Ã., \& Hinrikus, H. (2013). EEG Spectral Asymmetry Index for detection of depression at Individual and Fixed Frequency Bands World Congress on Medical Physics and Biomedical Engineering (Vol. 39, pp. 589-592): Springer Berlin Heidelberg

[19] Sullivan, M. (2004). Statistics: Informed Decisions Using Data. New Jersey: Pearson Education, Inc.

[20] Teplan, M., Krakovská, A., \& Štolc, S. (2009). EEG Characterization of Psycho-Physiological Rest and Relaxation. Paper presented at the Proceedings of 7th International Conference on Measurement, Bratislava.

[21] Tomkins, C., \& Hall, C. (2006). An introduction to non-parametric statistics for health scientists. University of Alberta Health Sciences Journal, 3(1), 20-26.

[22] Vega, C. F., \& Fernandez, F. J. R. (2012a, 9-11 Jan. 2012). Phase synchrony analysis of gamma band as tools to discriminate cognitive tasks. Paper presented at the Biosignals and Biorobotics Conference

[23] Vega, C. F., \& Fernandez, F. J. R. (2012b, 9-11 Jan. 2012). Recognition of mental task with the analysis of long-range temporal correlations on EEG brain oscillation. Paper presented at the Biosignals and Biorobotics Conference.

[24] Wen-Yuan, L., Chun-Jing, X., Bao-Wen, W., Yan, S., \& Shu-Fen, F. (2003, 2-5 Nov. 2003). Study on combining subtractive clustering with fuzzy c-means clustering. Paper presented at the Machine Learning and Cybernetics, 2003 International Conference on.

[25] Wilcoxon, F. (1945). Individual Comparisons by Ranking Methods. Biometrics Bulletin, 1(6), 80-83.

[26] Wilcoxon, F. (1947). Probability tables for individual comparisons by ranking methods. Biometrics, 3(3), 119122. 